\title{
Case Study New design 3 Blades Type on 35 Developed Area and Testing cavitation of Tailing Thai Boat
}

\author{
Jaruphant Noosomton $^{\mathrm{a} *}$, Jarruwat Charoensuk ${ }^{\mathrm{a}}$ \\ ${ }^{a}$ Faculty of Engineering, Department of Mechanical Engineering, King Mongkut's Institute of Technology Ladkrabang, \\ Chalongkrung rd., Ladkrabang, Bangkok Thailand 10520 \\ *Corresponding Author: noosint2514@ hotmail.com
}

\begin{abstract}
This paper presents to case study new design of propeller 3 blade types and focus cavitation flow around ship propellers experimentally. Testing of different types new model propellers and local type propellers are performed in this study. A 3-blades, skew angle 22 degree, developed area 35 which call Model-A (B3-22-35) and comparison which local type propeller commonly used by Thai fishermen. A practical design method was achieved by combining a vortex lattice lifting method and lifting surface method. The optimum circulation distribution yielding the maximum lift-to-torque ratio was computed for given thrust and chord lengths along the radius of the propeller by dividing the blade into a number of panels extending from hub to tip. The radial distribution of bound circulation could be computed by a set of constant-strength vortex elements. The cavitation phenomenon of new design appeared to be less than local type.
\end{abstract}

Keywords: Skew Angle, Developed Area, Cavitation Number $(\sigma)$.

\section{Introduction}

\subsection{Background of the Problem}

Cavitation flows are highly complicate because it is a rapid phase change phenomenon, which often occurs in the high-speed or rotating fluid machineries. It is well known that the cavitation flows is of great consideration in the design process of marine propellers and must be optimized for specified operating condition. Although computational fluid dynamics has played an important role in design process of marine propellers for more than two decades, a conventional design method so-called "The lifting line method (LLM)" and "A vortex-lattice method (VLM)" was introduced for the analysis of fully wetted propeller flows by G.Kuiper ${ }^{(1)}$, the method was later extended to treat unsteady sheer cavitating flows by Akihisa Konno ${ }^{(2)}$ and Breslin and colleagues ${ }^{(3)}$. In Kinnas ${ }^{(4)}$, Kinnas and $\mathrm{Pyo}^{(5)}$, a leading-edge correction was introduced to account for the defect of the linear cavity solution near a round leading edge, with this design method, the blade shape is replaced by an infinitely thin surface which takes the form of the blade camber line upon which a distribution of vortices is placed in both the spanwise and chord directions ${ }^{(8,9)}$. The method of this type used this basis for their formulations and the solution of the flow in many ways of the thin blades. Additionally, the vortex lattice method is adopted as part of the lifting surface method. It is discretize from the continuous distributions of vortices and sources which are replaced by a finite set of straight line elements of constant strength whose end points lie on the blade camber surface. From this system of line vortices, the velocities are computed at a number of suitably located control points between the elements. Their distributions at time and space have to be determined using the boundary conditions of the flow condition being analyzed. The source distributions, however, are independent with time and their distributions over the blade are established using a strip-wise application of thin blade at each of the radial positions.

When applied for the design of blade, the geometry of the blade is only partially known in so far as the radial distributions of chord, while rake, skew and section thickness distributions are known. The radial distribution of 
pitch and the chord-wise and radial distribution of camber remain to be determined. In order to solve the new design the source and vortex distributions representing the blades and their wakes need to be placed on suitable reference surfaces to enable the induced velocity field. Linear theory assumes that the perturbation velocities due to the propeller are small compared with the inflow velocities. However, the real viscous and turbulent behavior of flow shows the phenomenon of divergence with this design method, for instance, in the calculation of the radial loading parameter. If this carry a stage further the divergence becomes increasingly larger when using this method for determination of camber and angle of attack and hence section pitch. This is in part due to the variety of correction procedure adopted for the lifting line method which, a conceptual way, the basis of the lifting surface method ${ }^{(6,7)}$.

Despite of above mentioned, the methodology for low-speed propeller as normally used in shallow fishery in developing world has, however, been at the early stage and far from those normally performed in design and analysis of propeller blade in marine industry. This paper aims to show technique of vortex lattice lifting method and lifting surface method which have not yet been used for low-speed, diesel-engine driven, propellers for tailing Thai boat.

In this paper, the cavitation performance and open water performance of Tailing Thai Boat, 3 Blade type on 22 degree skew angle and 35 developed area. The effect of non-condensable gas mass fraction of predicted cavitation performance was mainly studied. The predicted cavity shapes were in good agreement with comparison the local domestic propeller. Which the shallow-fishery and tailing Thai boat used low-diesel engine by the researcher observation and result on open literature.

\section{Description and Experiment}

\subsection{Description}

\subsubsection{Propeller Design}

It's my intention to discuss these new developments. The focus of this paper is to assessment the supplication to propeller design and the possibilities which arise. For that purpose I will consider a local propeller designer, who has to design a ship propeller for tailing Thai boat. Which this researches to practical design method was achieved by combining a vortex lattice lifting method and lifting surface method.

\subsubsection{Propeller Performance}

The propeller design generally starts with a given hull form a required ship speed. Important goals of the propeller design are minimum power or call optimum efficiency and the correct rotation rate of the propeller by up to the main engine. And there are two important boundary conditions at full scale ${ }^{(6,7)}$.

\section{A. Determination of propeller inflow}

The propeller thrust is difficult to measure at full scale, but at least it is an existing force. This is different for the nominal wake distribution, which the full scale of source code program $(\mathrm{SCP})^{(10)}$ for calculations not only gives the required propeller thrust, but also the total velocity distribution in front of the propeller. And this total velocity field in front of a propeller can be validated at full scale because it exists in this SCP. The total inflow into the propeller induced velocities can be subtracted to give the inflow distribution for the propeller design. The propeller induced velocities can be estimated from a preliminary propeller design and can be adjusted iteratively later on. When a calculation for model scale is combined with a propeller performance calculation the calculated required torque at model scale can be validated also by a propulsion test, which gives another anchorpoint for the calculation of the propeller inflow distribution. Again, this gives only an anchorpoint. The calculation for full is fully SCP. An important aspect of this approach is that the nominal wake is again omitted.

\section{B. Determination of Thrust}

The propeller design then has to find the required thrust and inflow velocity or wake distribution of propeller. What the normally gets from model test is the resistance, a nominal wake field and a thrust deduction. A rather crude extrapolation is then applied involving flat plate resistance and roughness allowances to arrive at a resistance prediction for full scale. The problem is that the full scale resistance cannot be measured. It is a purely conceptual quantity to arrive at the required full scale thrust. It seems attractive to validate the source code program (SCP) for calculations with the measured resistance at model scale. This resistance is measured with high accuracy because even very small differences count for the fuel consumption of a ship. As a result the source code program (SCP) for calculations sometimes cannot match the accuracy, however, is a wrong comparison, because there is not real 
need for the source code program (SCP) for calculations to predicted the model resistance more accurately than the whole chain in the experimental extrapolation method. And this can be include the propeller action, although a realistic loading distribution makes symmetry of the calculation impossible and thus increases the calculate time. The challenge of research program at model basins is then not to calculate the ship resistance at model scale with the same accuracy as the model test, but to establish a correlation between the calculated results of propeller torque and full scale data. If the deviations of the full scale torque from the calculated torque are smaller than from extrapolated model tests, an improvement has been obtained. In that case the resistance without propeller and the nominal wake become irrelevant.

\subsection{Experimentation}

From the design method mentioned in the earlier section the characteristic curves under cavitation and non-cavitation conditions can be determined. Usually, the characteristic curves are variations of thrust and torque coefficients, with respect to the advance coefficient. In obtaining the characteristic curves by lift surface method the rotational speed was kept constant and the advance velocity was varied in the range of allowable water tunnel flow speed (e.g., in the current water tunnel, $0-3.5 \mathrm{~m} / \mathrm{s}$ ). Thus, different values for the advance coefficient were obtained, see Fig. (1). this section also illustrates the test results of newly designed propellers, namely Model A and against the conventional propeller, Model $\mathrm{C}$. The main purpose of this performance test on this newly designed propeller in the water tunnel against the domestic type conventionally used in Thailand (Table1) was to compare the behavior of occurring bubble at various rotational speeds. In the non-cavity test, the static pressure was constant at the value of the usual operating pressure.

Table 1. Principal particular of new design Model-A and

\begin{tabular}{|l|c|c|}
\multicolumn{1}{|c}{ Model-C (Local type) } \\
\hline Number of blades & $\begin{array}{c}\text { Model-A } \\
(3-22-35)\end{array}$ & $\begin{array}{c}\text { Model-C } \\
\text { (Local) }\end{array}$ \\
\hline Diameter (m) & 0.24 & 2 \\
\hline P/D & 0.94 & - \\
\hline EAR=AE/AO & 0.35 & $\sim 0.50$ \\
\hline Skew Angle (degree) & 22 & $\sim 21$ \\
\hline Open W. eff. & 0.83 & - \\
\hline
\end{tabular}

\begin{tabular}{|l|l|l|}
\hline Cavity at $0.7 \mathrm{R}$ & 1.566 & - \\
\hline
\end{tabular}

The reason behind the above mentioned procedure lies in the type of dimensional analysis that is used for deriving non-dimensional coefficients in characteristic curves. The necessary dimensional analysis for open water conditions has been calculated but not given in this paper. In cavitation analysis, two kinds of curves were derived. One was the characteristic curve in a constant cavitation number, which was used to show the deviation of torque and thrust from a non-cavitation state. The second was the diagram of torque and thrust coefficients on the basis of the cavitation number, which was used to show the cavitation breakdown analysis. Then the static pressure and advance velocity inside the cavitation tunnel test section were lowered gradually in order to sketch the required diagrams. Axial velocity was varied from 0.3 to $3.5 \mathrm{~m} / \mathrm{s}$. It is seen from Fig. (1) That at low values of $J$, there is an appreciable difference between the results. This is a general fact because according to the definition, $K_{T}$ is proportional to the first power of thrust rather than the second power of rotational speed as seen in the denominator.

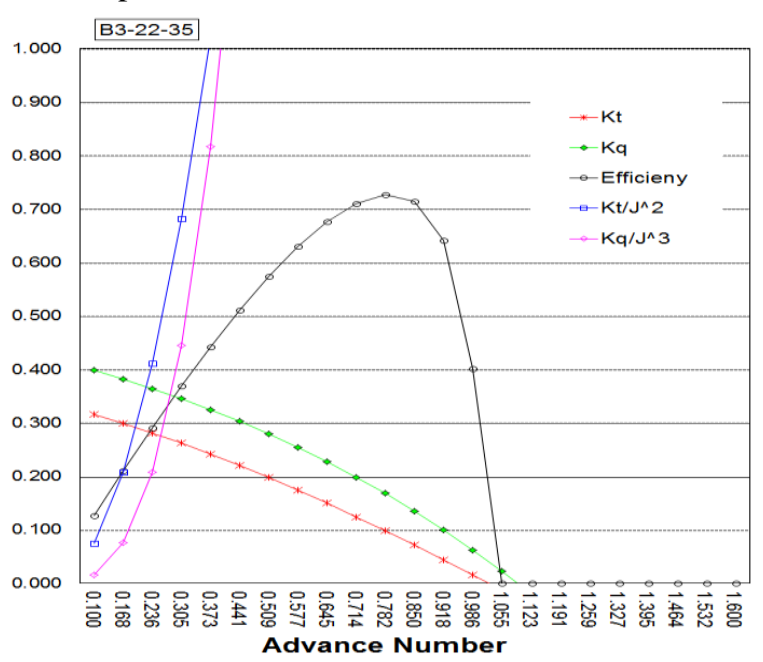

Fig. 1. Characteristic curve of Model-A Type 3 Blade $22^{\circ}$ skew angle and 35 developed area (B3-22-35)

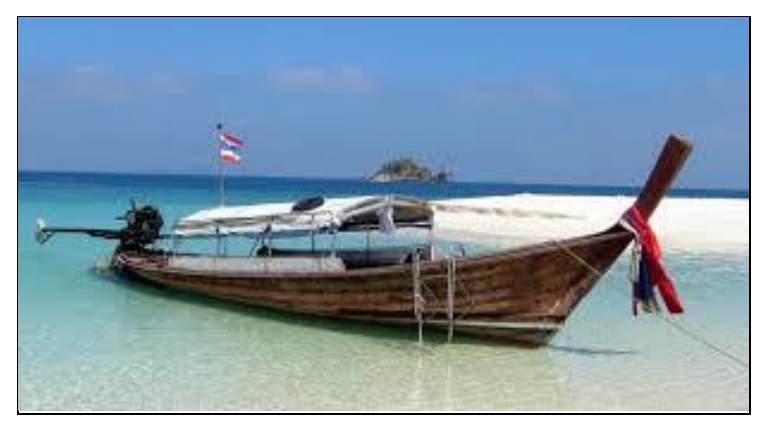

Fig. 2. Illustrations of Tailing Thai Boat with 2-blades type propeller 


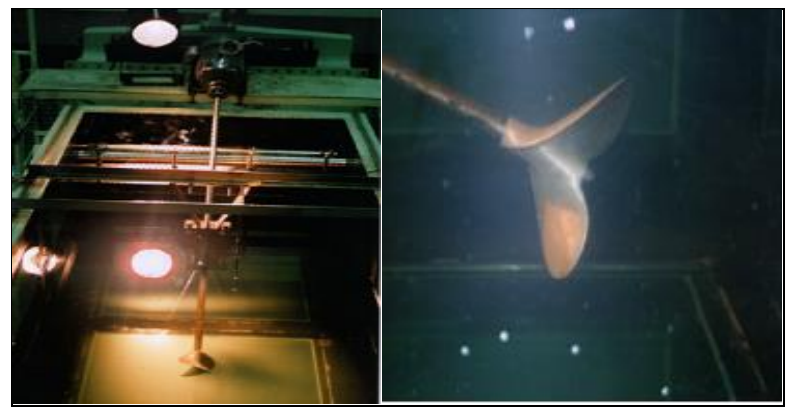

Test unit and propeller installation in a water tunnel laboratory in the southern part of Thailand. Fig. (2) Depicts the installation of the propeller on tailing Thai Boat and Fig. (3) Shows the installation of the propeller inside the water tunnel. Figs. (4) and (5) Shows the body plan of the geometry and propeller model of the Model A [B3-22-35].

Fig. 3. Installation of propeller in the water tunnel
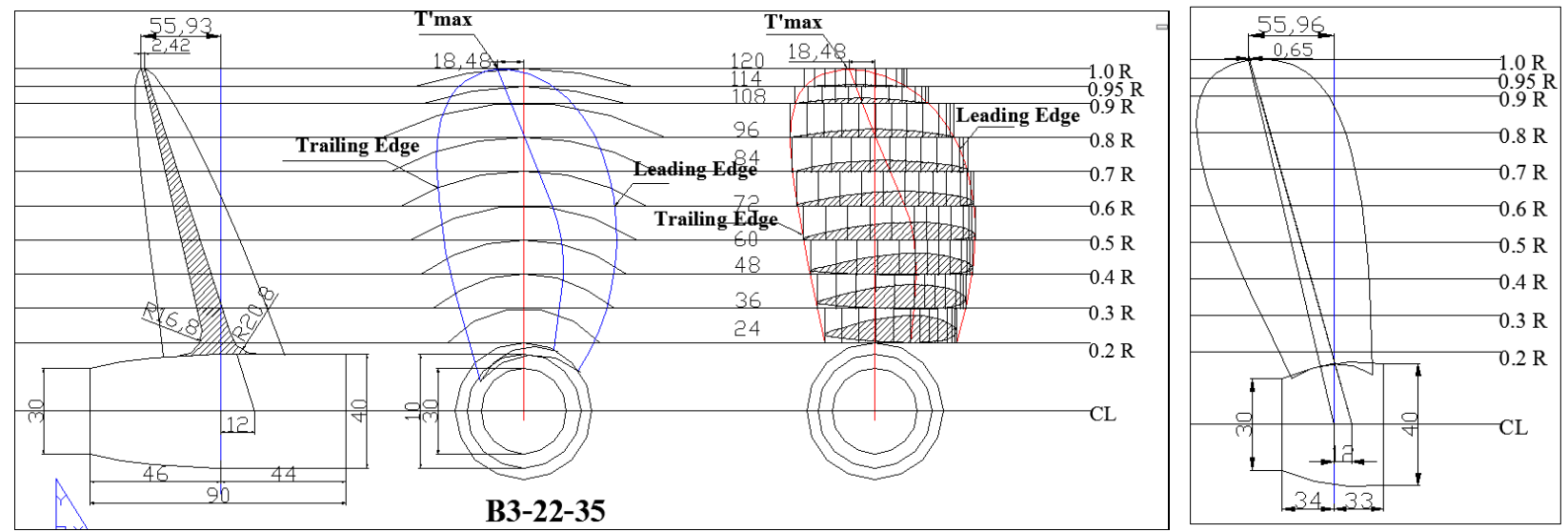

Fig. 4. Drawing Design 3 Blades Type $22^{\circ}$ Skew Angle Developed Area 35(New Design, Model-A)

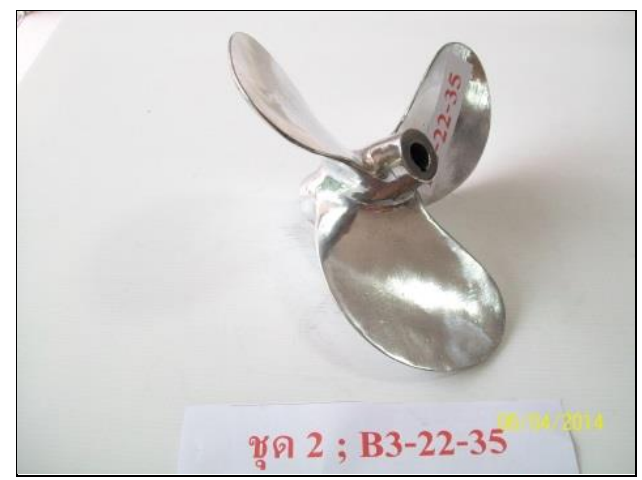

Fig. 5. Illustration of propeller Model Type B3-22-35 (New Design, Model A)

The test results of model $\mathrm{A}$ and model $\mathrm{C}$ in non-cavitation states with the rotational speeds of $850 \mathrm{rpm}$ and $900 \mathrm{rpm}$ are depicted in Figs. (6),(7) and (8). The testing results of propellers for non-cavitation conditions are essential before proceeding to cavitation conditions for two reasons. Firstly, this test result will be a platform where the numerical method is validated for simulation of the fluid flow around the propeller models. Secondly, solving the flow fields in non-cavitating conditions is used as the initial condition for cavitation simulations which will be presented in the next paper.
The characteristic curve throughout cavitation and cavitation free conditions are also compared, see Fig. (1) For a blade designed up to maximum rotational speed of $1185 \mathrm{rpm}$ (a predefined designed speed) when maximum cavitation occur. Due to limitation of test tank facility, operating condition was carried out $850 \mathrm{rpm}$. These data were used as a design condition for the new models and therefore used to carry out the experimental result. The misbelieving among local operators regarding the role of cavitation bubbles on performance of the propeller is the major cause of inefficient use of energy in Thailand's local fishery industry. It is necessary to provide strong evidence, how generated bubbles could deteriorate the blade's mechanical integrity and to see how bubbles could have negative effect to the blade performance. To serve this purpose the test result on Model C was done at $850 \mathrm{rpm}$ and further extended to $900 \mathrm{rpm}$ in order to demonstrate full cavitation. According to the plan, this experimental work would include test conditions of Model A at $850 \mathrm{rpm}$. However, the support structure of the test tank and the bed of the channel had shown a sign of damage. The experimental work at this particular condition was then cancelled. 
Table 2. Summary of test conditions all experiments were carried out at water flow speed of $3.5 \mathrm{~m} / \mathrm{s}$.

\begin{tabular}{|c|c|c|l|}
\hline $\begin{array}{c}\text { Test } \\
\text { condition,(rpm) }\end{array}$ & Model A & Model C & Remark \\
\hline 850 & $\checkmark$ & $\checkmark$ & To compare cavitation among Model A and C \\
\hline 900 & N/A** & $\checkmark$ & To observe full cavitation of Model C \\
\hline
\end{tabular}

** Not available due to high thrust force and bed pressure that may cause damage to the test tank

The quantitative investigation on cavitation had been carried out given in Fig. (6) As for model A. The angular speed of this test condition was at $850 \mathrm{rpm}$, this is a new design for Shallow-Fishery and Tailing Thai Boat. Below are series of pictures taken in consequence by a camera at the time impulsive force was detected.

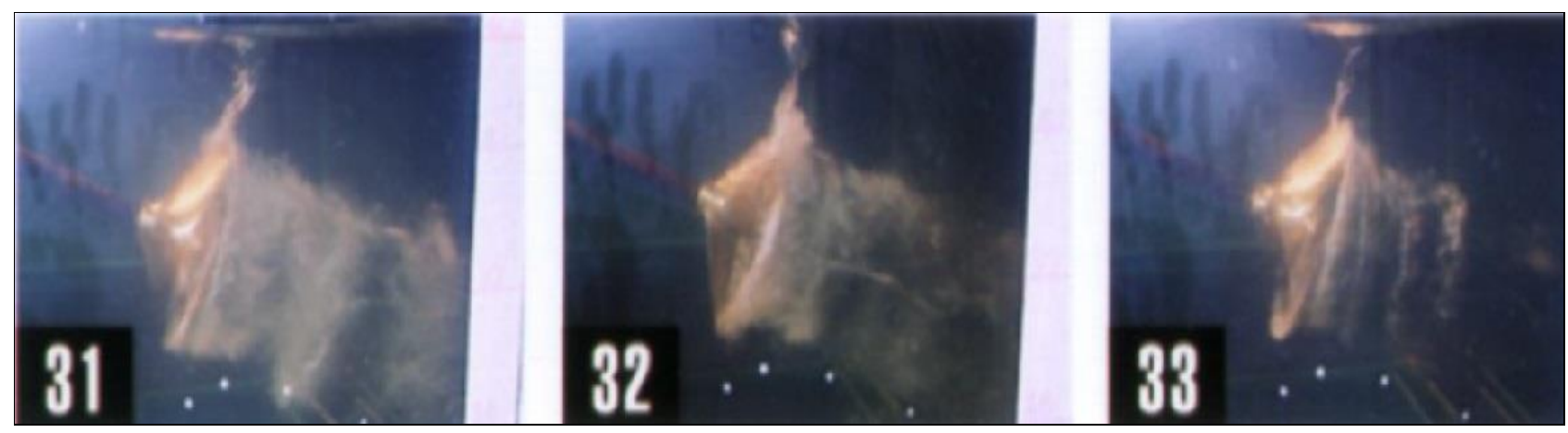

Fig. 6. Illustration of propeller cavitation Model A at rotational speed $850 \mathrm{rpm}$.

\section{Discussion}

\subsection{Discussion; Cavitation of Model A (New design) and Model B at $850 \mathrm{rpm}$.}

The rotational speed $850 \mathrm{rpm}$ from Fig. (6) found that skew angle $22^{\circ}$, developed area 35 have been that ring of cavitation less many than that occurred less than model $\mathrm{C}$. In Figs. (7) and (8) are the photographs for propeller type: Model C, which is commonly used by locals. They show the cavitation developed strongly when the rotational speed is as high as $850 \mathrm{rpm}$ which the pictures are found that the occurred of behavior cavitation in Model C, it is formed in front of blade (discharge side) and it a negative pressure. So the demonstrated seen how that the propeller was low performance.

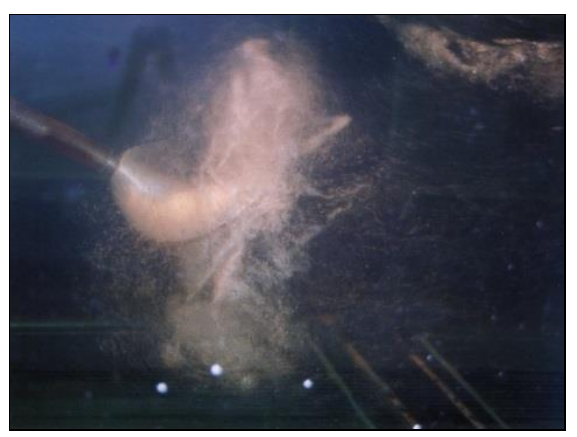

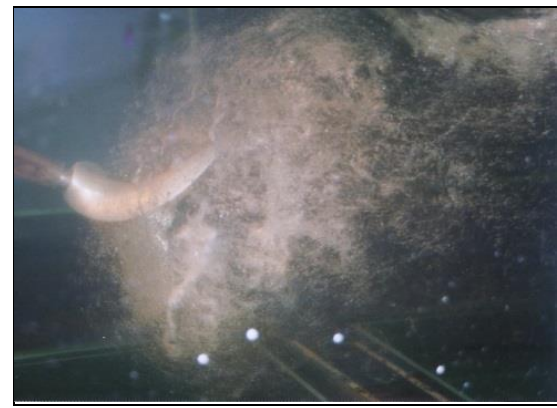

Fig. 7. Illustration of propeller cavitation Model C at rotational speed $850 \mathrm{rpm}$

One should be noted that in Fig. (7) Cavitation occurred with Model C at $850 \mathrm{rpm}$ and at axial velocity of $2.5 \mathrm{~m} / \mathrm{s}$. It suggested the cavitation surface was glassy smooth and the propeller blade surface was seen clearly behind the surface. Thus, it could be deduced that the formed cavitation was the sheet cavitation. The propeller was further tested at 900 rpm with a cavity greater developed up to full area of the blade, the photographs of such test condition is given in Fig. (8) The large pressure fluctuation was measured twice in a series showing the bursting phenomenon in one rotation of propeller blade In the first stage, bursting tip vortex cavitation from the preceding blade interfere the sheet cavity, and that made the sheet cavity unstable and its trailing edge swelled significantly. The sheer cavity was shed to the tip vortex cavitation and a vortex cavity from the trailing edge of the sheer cavity intertwined with each 
other, causing implosion and rebound again and again. At this time, the tip vortex cavitation was spitted into many bubbles, and a bubble cluster, including small vortex cavities, formed. Instantaneously, bursting occurred when the following blade approached upstream of the vibrating vortex, which had been intensely disturbed in the first bursting. The following blade swiftly obstructed the flow and caused tip vortex cavitation implosion and rebound again, and at that time bubbles in the tip vortex cavitation collapsed repeatedly and randomly.
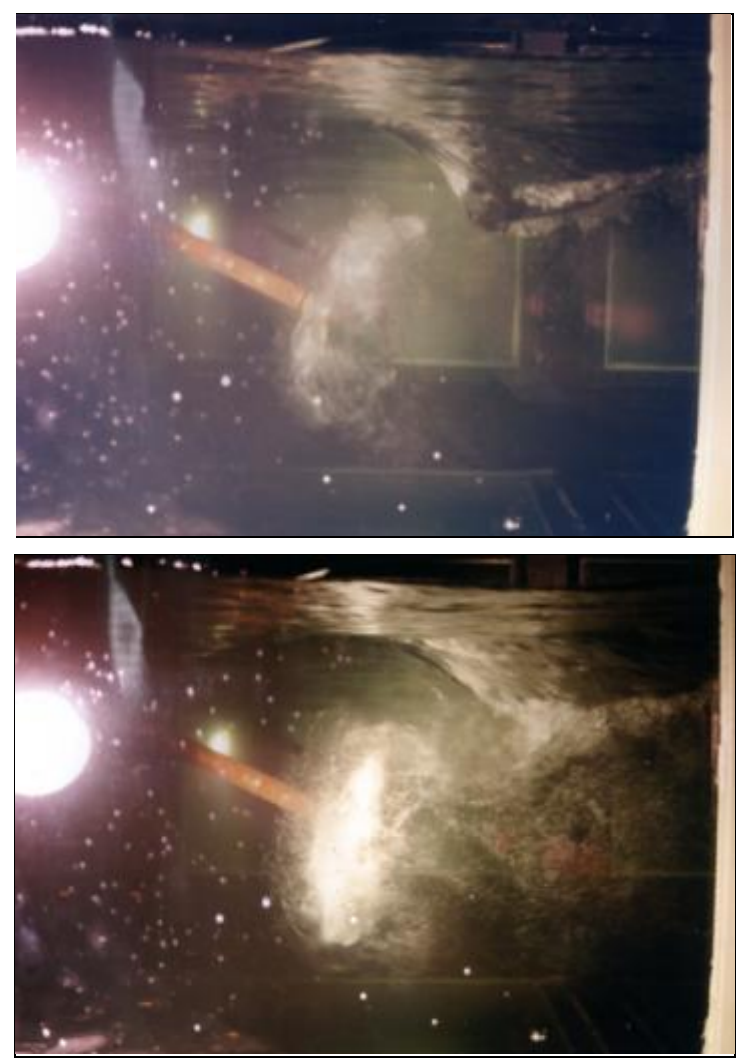

Fig. 8. (a) Initial Cavitation of Model C

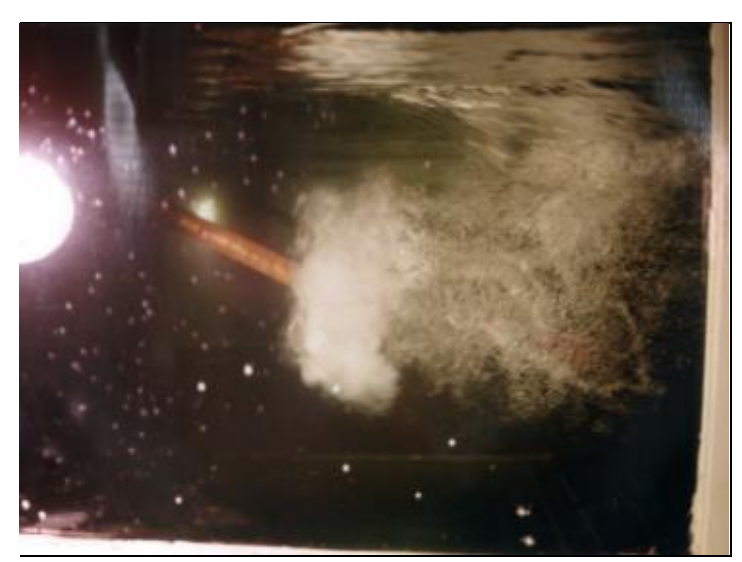

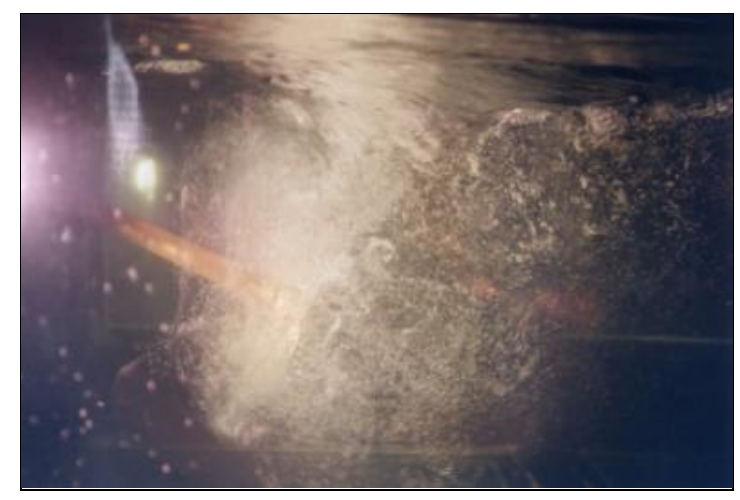

Fig. 8. (b) Full cavitation at rotational speed $900 \mathrm{rpm}$, local type propeller

\section{Conclusion}

In this paper, cavitation flow is investigated experimentally on new designed and one conventional propeller for shallow-fishery and tailing Thai boat. The preliminary experimental result of the new blades shows better performance when compared with local domestic type. The cavitation performance and open water performance of Shallow-Fishery and Tailing Thai Boat based on transport equation and standard $k-\omega$ turbulent. The elucidation bursting phenomenon of tip vortex cavitation, with experiments in a water tunnel were carried out with two propellers, new propeller (B3-22-35) having different thrust coefficients and cavitation numbers. The results obtained suggested that, the modification in blade design has been considered to reduce the fluctuating pressure caused by bursting. One is to stabilize the tip vortex cavitation, and the other is to reduce the cavitation itself. The occurrence of tip vortex cavitation can be predicted by the rate of change of the tip vortex intensity. The bursting occurs because the rate of change of the tip vortex intensity is related to the stability of the tip vortex. The achievement of this investigation will be presented in the next publication. However, this investigation suggests that the present approach is practicable as a design approach for low speed propeller for Thailand fishery industry.

\section{Acknowledgement}

This study was partly supported by the Thailand Research Fund (TRF; www.trf.or.th) and the authors would like to thank Nakon Si Thammarat Seaboard Industrial College, and Captain Sarawut Wongchenyour (RTN.), Royal Thai Navy for contributions toward this work. 


\section{References}

(1) G. Kuiper: "New developments and propeller design", Journal of Hydrodynamics, Ser. B, Volume 22, Issue 5, Supplement 1, pp.7-16, 2010

(2) Akihisa Konno et.al.: "On the mechanism of the bursting phenomena of propeller tip vortex cavitation", Journal of Marine Science and Technology, Volume 6, Issue 4, pp. 181-192, 2002

(3) Breslin J., Van Houtec R., Kerwin J., and Johnson C,-A.: "The cortical and experimental propeller-induced hull pressures arising for intermittent blade cavitation, loading, and thickness", Transaction of SNAME, 90:111-151,1982

(4) Kinnas S.: "Leading edge corrections to the linear theory of partially cavitation hydrofoils", Journal of Ship Research, 35:15-27, 1991

(5) Kinnas S., and Pyo S.: "Cavitation propeller analysis including the effects of wake alignment", Journal of Ship Research, 43:38-47, 1992

(6) G Kuiper, M Grimm, B McNeice, et.al.: "Propeller Inflow at Full Scale during a Manoeuvre", 24th Symp. On naval Hydrodynamics, Kuruoka, Japan, 2002

(7) G Kuiper, S D Jessup.: "A Propeller Design Method for Unsteady Conditions", SNAME Transactions, Centennial Meeting, 1993

(8) Otsuka S., Tsujimoto Y., Kamijo K., Furuya O.: "Frequency Dependence of mass Flow Fain Factor and Cavitation Complicance of Cavitating Inducers", ASME Journal of Fluid Engineering, Vol.118, No.4, pp.400-408, 1996.

(9) Rhee S.H., Kawamura T. and Li H.: "Propeller cavitation study using an unstructured grid based Navier-Stoker solver", ASME Journal Fluids Engineering, Vol.127, pp.986-994, 2005

(10) J Noosomton, J Charoensuk: "Performance Design and Testing of Propeller for Shallow-Fishery and Tailing Thai Boat: 2-blade type Part (2) : A method for optimum cavitation of ship propeller", [C] $4^{\text {th }}$ TSME International Conference on Mechanical Engineering, Pattaya Chonburi, 16-18 October 2013 